INSTITUT NATIONAL DE RECHERCHE EN INFORMATIQUE ET EN AUTOMATIQUE

\title{
Energy and Throughput Optimization for Relay Based Heterogeneous Networks
}

\author{
Anis Ouni - Ahmed Saadani — Hervé Rivano
}

\section{$\mathbf{N}^{\circ} \mathbf{7 7 3 0}$}

September 2013

\section{apport}

de recherche 



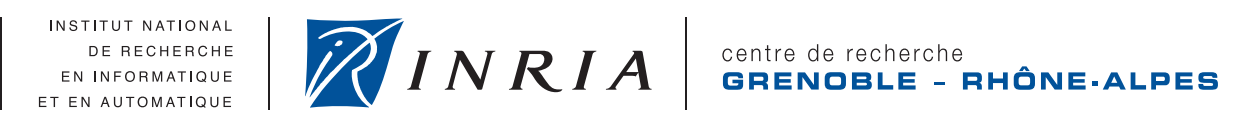

\title{
Energy and Throughput Optimization for Relay Based Heterogeneous Networks
}

\author{
Anis Oun调, Ahmed Saadan用, Hervé Rivand $\$$ \\ Thème : 1 \\ Équipes-Projets Urbanet \\ Rapport de recherche $n^{\circ} 7730$ - September 2013 - 16 pages
}

\begin{abstract}
High data rate is a challenge for the next generation cellular networks. This objective needs a significant densification of relay nodes within macro cell. In the LTE-Advanced network, multi-hop relaying has been taken as a promising key technique to provide high throughput to the users and to improve the area coverage. Besides, minimizing the energy consumption and electromagnetic pollution is an economic challenge for the operators. This paper is focused on relay based heterogeneous cellular network like LTE-Advanced. We investigate the problem of throughput and energy consumption optimization. Our contributions are two-fold. First, we develop an optimization tool to calculate an optimal offline configuration of heterogeneous cellular network that maximizes the network capacity with low energy consumption. Second, we highlight a significant gain due to the deployment of relay nodes and we investigate the energy-capacity tradeoff.
\end{abstract}

Key-words: LTE-Advanced, multi-hop relaying, capacity, energy consumption, tradeoff, optimization tools.

\footnotetext{
* Corresponding author - Partially supported by ANR VERSO ECOSCells

† Université de Lyon, INRIA, INSA Lyon, CITI, F-69621, France

$\ddagger$ Orange Labs, Issy-les-Moulineaux 92794, France

$\S$ Université de Lyon, INRIA, INSA Lyon, CITI, F-69621, France
}

Centre de recherche INRIA Grenoble - Rhône-Alpes 655, avenue de l'Europe, 38334 Montbonnot Saint Ismier Téléphone : +33476615200-Télécopie +33476615252 


\section{Optimisation de la la consommation d'énergie et de débit des réseaux cellulaires hétérogènes}

Résumé : Offrir un haut débit avec une consommation d'énergie faible est un défi pour les réseaux hétérogènes cellulaire comme LTE-Advanced. Ce papier se focalise sur l'optimisation de la consommation d'énergie et le débit de ces types de réseaux. Nous proposons deux contributions principales. La première contribution est de développer des modèles d'optimisations efficaces pour calculer une configuration optimale des réseaux cellulaires hétérogènes qui maximise la capacité du réseau à faible consommation d'énergie. La deuxième contributions et de quantifier le gain en énergie et en débit dû au déploiement des nœuds relais, et d'étudier le compromis débit-énergie.

Mots-clés : Réseaux Radio Maillés, capacité, consommation énergétique, allocation de ressource. 


\section{Introduction}

The 3GPP LTE-Advanced is one of the most efficient and flexible communication standards. It has emerged as a promising technology for next generation cellular networks to support high data rate requirements and increase the capacity provided to users, e.g. for meeting the requirements of mobile multimedia applications. However, the rapid growth of traffic load generated by the mobile terminals is accompanied by an unsustainable increase of energy consumption: in a cellular network, about $80 \%$ of the energy is consumed by the access network 1. Because of increased electricity costs and electromagnetic pollution in the society, the operators are interested in reducing the energy consumption. Consequently, many research projects have focused on the optimization of throughput and energy consumption in mobile systems [2, 3. In this paper, we assume an LTE Advanced Heterogeneous Networks composed of a twofold architecture [4,5. On the one hand a wireless backhaul interconnects small base stations (Relay Node, a.k.a RN) with a macro base station (a.k.a. eNB). On the other hand, user equipment (UE) is either connected to the macro base station in the access link, or to the relay node (Fig. 1). The eNB is equipped with a tri-sector antenna that can transmit at distance between a few hundred meters to a few kilometers. The RNs have significantly lower transmit power compared to the eNodeB and are equipped with omnidirectional antenna to cover a few tens of meters. RNs are mainly deployed to extend the coverage of macro cell or to increase the users throughput in areas with dense data usage.

Improving the energy consumption or the throughput of cellular network has received considerable attention in recent years [6, 1, 7], but only very few papers investigated jointly these two problems or the tradeoff between them. The main objective of this paper is to address the optimization of both network capacity and energy consumption. Network capacity is defined as the the maximum achievable total throughput in the macro cell under a fairness criteria. Improving the network capacity is necessary for providing a better quality of service to a larger number of users. Our notion of the energy consumption is the overall energy consumption in the network of eNBs and RNs.

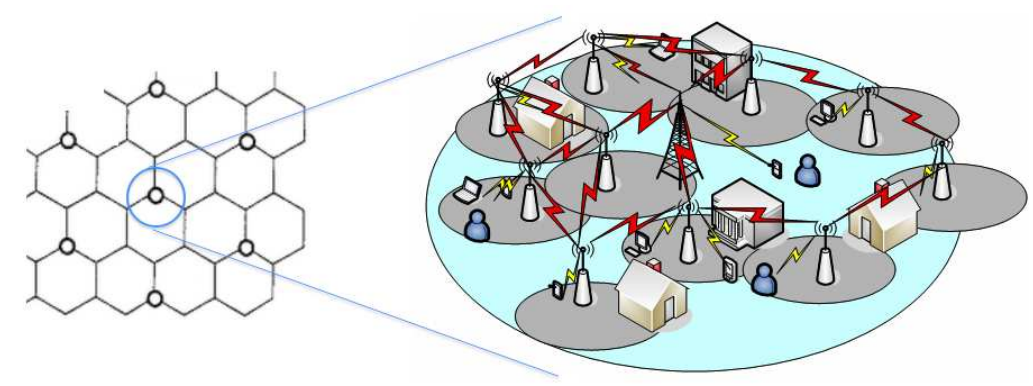

Figure 1: Relay based network like LTE-Advanced: user equipment (UE) is either attached to the macro base station (eNB) in the access link, or to the relay node $(\mathrm{RN})$.

The contributions of this paper are two-fold. First, we develop an optimization framework on the basis of a previous work for wireless mesh networks 8 . This tool is dedicated to the study of the capacity and the energy consump- 
tion of heterogeneous cellular networks with multi-rate and continuous power control. Second, the gain of network capacity and energy consumption due to the deployment of relay nodes is quantified, and the energy-capacity tradeoff is investigated.

The remainder of this paper is organized as follows. In Section 2 we present the related work. Section 3 describes the general system model and presents our optimization framework. In Section 4, we investigate the gain brought by relay nodes and the tradeoff between energy consumption and capacity. The last section concludes our work and gives future directions.

\section{Related work}

Improving the energy consumption or the throughput has been studied extensively in the heterogeneous network context. However, these two problems are not usually studied jointly. 9 focused to calculate a realistic achievable network throughput of multi-hop relaying cellular networks. An optimization problem is formulated in order to find the best schedule of transmission schemes to maximize the network throughput subject to different constraints.

[10] provided a first analysis of the energy efficiency of two relaying schemes. This work highlighted a first indication of the potentiality of relay nodes to save energy consumption. However, the authors have considered a small scale simulations in a multi-cell environment, in full load conditions and by measuring only the radiated power. In 11, the energy efficiency of different small node deployments, namely relay nodes and Picocells, has been evaluated within the 3GPP LTE-Advanced framework. Simulation results on the uplink and the downlink of LTE-Advanced networks have showed a decrease in the area power consumption $(\mathrm{APC})^{1}$ required to achieve a certain coverage criterion and an increase in the throughput power consumption (in bit-per-power unit) in a predefined coverage area for Picocell deployments; RN deployments offer a similar performance to macrocells.

In [8], a multi-objective framework has been developed to study the tradeoff between capacity and energy consumption of wireless mesh network. This framework uses a single rate transmission and a Signal-to-Noise-and-InterferenceRatio (SINR) based model. This work is based on a trend of optimization techniques: a column generation algorithm isolates the routing and scheduling models from the computation of concurrent links activations, and the computation of time/frequency resource allocation accounting for the energy expenditure. In 12, 7, the authors have formulated the joint resource allocation, user association and reuse pattern problem in the context of throughput optimization of heterogeneous network. This model does not use a continuous power control and tri-sector antenna for macro-cell.

\footnotetext{
${ }^{1} \mathrm{APC}$ assesses the power consumption of a network relative to its area and is measured in kilowatt per square kilometer.
} 


\section{System model and Optimization framework}

\subsection{Problem definition and assumptions}

We consider a heterogeneous cellular network characterized by multiple tiers of base-stations where a set of relay nodes are deployed within macro cells. The eNB and RN are equipped, respectively, with tri-sector and omni-directional antennas.

The LTE frame is divided in 20 time-slots where the duration of one timeslot is $0.5 \mathrm{~ms}$. Two adjacent time-slots are grouped into a sub-frame of length 1 ms, corresponding to a Transmission Time Interval (TTI). Each time-slot corresponds to 7 OFDM symbols, which are preceded by a cyclic prefix to avoid inter-symbol interference. The bandwidth corresponding to a slot (7 OFDM symbols) is subdivided into several blocks of 12 subcarriers, each of which is called a Physical Resource Block (PRB). The smallest resource unit that can be allocated to a user covers a TTI of $1 \mathrm{~ms}$ and a PRB (bandwidth of $180 \mathrm{khz}$ ), called a Scheduling Block (fig. 2). In each scheduling block, a node can adapt its

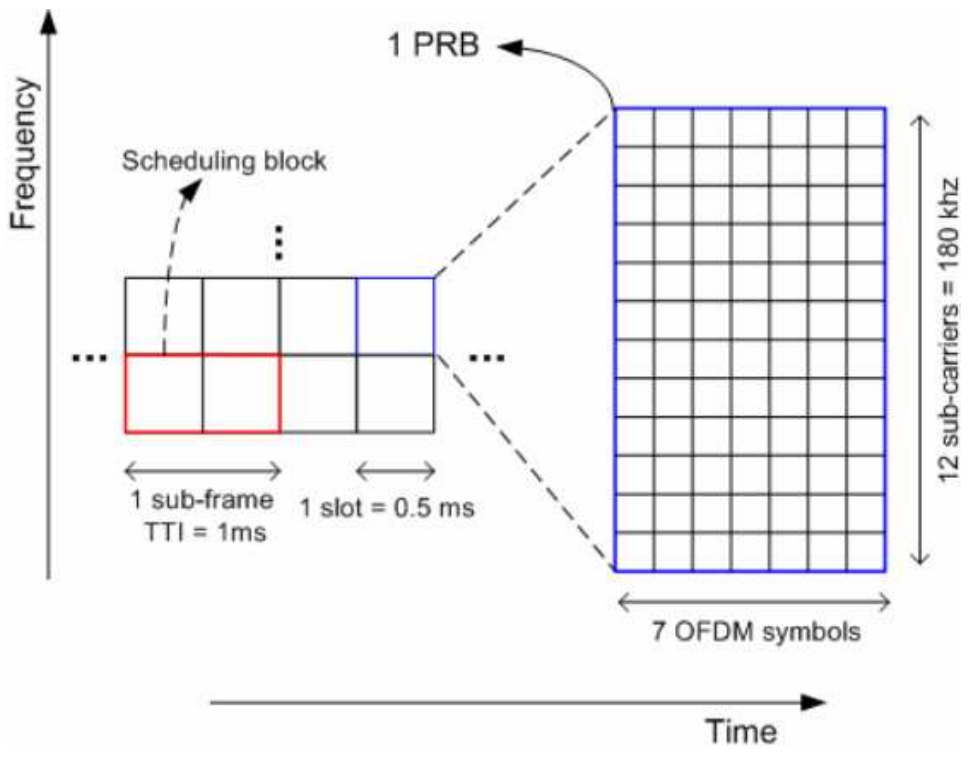

Figure 2: An illustration of resource element in LTE

transmission rate (associated to a Modulation and Coding Scheme, MCS) and adjust continuously its transmission power. We assume that the channel gains are independent from the PRB, but the optimization models that we present further on are generic and can take into account the case where the channel gains are different for different channels. Each RN can transmit and receive, simultaneously, on multiple different PRB. For each UE, our framework resolves jointly a routing and scheduling problem which consists in calculating the optimal path between the UE and eNB, and the optimal number of scheduling blocks to be allocated to each node in order to send/receive its traffic. Recall that the route between a UE and the eNB can be alternatively direct link or a multi-hop path through RNs. 


\subsection{Energy consumption model}

The energy consumption model is based on the model proposed in the EARTH project 13,14$]$. A base station, $u(\mathrm{eNB}$ or $\mathrm{RN})$, can be in a non-operational or in an operational state. In a non-operational state, some components are always on (due to signal processing, battery backup, as well as site cooling) and they consume a given quantity of power denoted $P_{0}$. In an operational state, we assume two modes, reception and transmission, which consume, respectively, $\left(P_{0}(u)+\Delta_{p}(u) \cdot P_{t}(u)\right)$ and $\left(P_{0}(u)+P_{r}(u)\right)$. The coefficient $\Delta_{p}$ accounts for the power consumption that scales with the average radiated power due to amplifier. In transmission mode, the energy consumption is near-linear with the transmission power $P_{t}(u)\left[14\right.$. The power consumed in reception mode, $P_{r}(u)$, is estimated based on actual measurement of various references presented in [13]. Since the energy consumption of an user equipment is extremely lower than that of a base station, we assume that it does not exceed 1.0 Watt independently of its state (standby, operational) [14]. The main parameters are summarized in Table 1 .

Table 1: Consumption power model parameters

\begin{tabular}{|c|c|c|c|}
\hline BS type & $P_{\max }[W]$ & $P_{0}[W]$ & $\Delta_{p}$ \\
\hline \hline $\mathrm{eNB}$ & 40 & 712 & 14.5 \\
\hline $\mathrm{RN}$ & 1 & 14.9 & 8.5 \\
\hline
\end{tabular}

\subsection{Network model and notations}

The heterogeneous cellular network can be modeled as a directed graph $\mathcal{G}$ composed of a set of nodes $V$ and directed links $\mathcal{L}$. The set $V$ of nodes is composed of three subsets, $V_{B S}, V_{R N}$ and $V_{U E}$ which represent, respectively, the set of eNBs, RNs and UEs. Each node eNB, RN and UE has a total transmit power equal, respectively, to $P_{e N B}, P_{R N}$ and $P_{U E}$. For each scheduling block, a node can transmit with a rate $r_{j} \in R, j \in\left[1, N_{r}\right]$ where $N_{r}$ is the rates number. The grid of resource blocks, illustrated by the Fig. 2, is modeled as follows. We assume that the time is divided into time slots and the bandwidth is partitioned into a set of orthogonal frequencies (PRB), denoted by $K_{f}$.

A link $l \in \mathcal{L}$ is identified by the following physical parameters:

- $o(l), d(l)$ : are, respectively, the origin and destination nodes of $l$.

- $k(l)$ : is the PRB used by the origin $o(l)$ to communicate with the destination $d(l), k \in K_{f}$.

- $P_{t}(o(l))$ : is the transmit power of the node $o(l)$. Recall that each node can continuously tune its transmit power at each transmission in order to reduce the interferences or to adapt the link quality. A node can be allowed to transmit at the same time on several frequencies. In this case, the maximum transmit power budget is shared between these transmissions: $\sum_{k} P_{t}^{k}(u) \leq P_{\max }$ 
- $r(l)$ : is the transmission rate, in bits per second, which depends particularly on the MCS and on the channel gain on the corresponding frequencies. It takes value from a set of available rates $R=\left\{r_{j}\right\}, N_{r}=|R|$. Without loss of generality, $0<r_{1}<r_{2}<\ldots<r_{N_{r}}, r_{1}$ is the lowest rate transmission.

A communication between any two nodes is successful, with a transmission rate $r$, only if the SINR at the receiver exceeds the minimum threshold $\beta(r)$. The SINR condition at the receiver, in the $\mathrm{PRB} k \in K_{f}$, and in the presence of a set of concurrent transmissions $s$ is expressed by the following equation:

$$
S I N R_{d(l)}=\frac{P_{t} \cdot G(l)}{\mu+\sum_{l^{\prime} \neq l, l^{\prime} \in s} P_{t}^{\prime} \cdot G\left(l^{\prime}\right)} \geq \beta(r),
$$

Where $\mu \in \mathbb{R}^{+}$represents the thermal noise at the receiver and $G(l)$ is the channel gain which takes into account the radio propagation model (path loss, fading and shadowing) and the antennas gains. Note that each antenna is characterized by an antenna radiation pattern based on a signal attenuation function $A$. For the omnidirectional antennas used by RNs, $A=0 d B$. For sector-antenna, used by eNBs, the antenna pattern is calculated using the following equation [15:

$$
A(\theta)=-\min \left[12 \cdot\left(\frac{\theta}{\theta_{3 d B}}\right)^{2}, A_{m}\right]
$$

where $\theta$ is the angle between the direction of interest and the antenna boresight, $\theta_{3 d B}=70^{\circ}$ is the $3 d B$ beamwidth and $A_{m}$ is the maximum attenuation.

Definition 1 (Conflict free scheduling) A set of transmissions is simultaneously feasible if and only if Eq. (1) holds at all receivers and $\forall l^{i}, l^{j} \in s, i \neq j$, $o\left(l^{i}\right) \neq o\left(l^{j}\right), d\left(l^{i}\right) \neq d\left(l^{j}\right)$ and $o\left(l^{i}\right) \neq d\left(l^{j}\right)$. We define an Independent Set (ISet) as a collection of simultaneously feasible transmissions. All the links in a ISet can be scheduled at the same TTI without creating any decoding conflict. Hence, by scheduling only ISets, we will make sure that the schedule is conflict free.

\subsection{Optimization tools}

Our optimization framework is mainly composed of two parts, as depicted in Fig. 3. The first part (called HetGen) is responsible to generate the network scenario parameters (e.g., the set of links, nodes, the channel gain between each pair nodes, etc.) based on 3GPP Specifications [15, 4,5]. This network scenario depends on the number of eNB, UEs and the environment (Urban or Rural). The UEs are randomly distributed with an Uniform process in each macro-cell while the RNs are deployed close to the cell borders where the SNR of eNB is low, an example of RNs and UEs deployment is depicted in Fig. 4. HetGen also provides the possibility of visualizing the network topology. The second part is based on a linear program and a column generation algorithm adapted from the one presented in [8]. This is implemented using AMPL and solved with CPLEX solver 16, 17. Finally, AMPL logs data to an output trace file, which contains all the needed data to evaluate the performance of the system. 


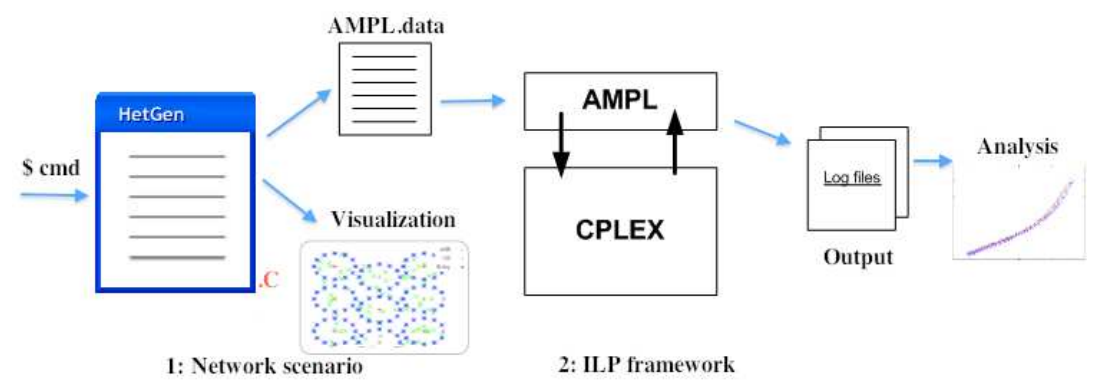

Figure 3: Complete flow of optimization tools.

This framework allows particularly to compute an optimal system setting of the network to maximize the capacity with low energy consumption or to minimize the energy consumption with higher network capacity ${ }^{2}$. We mean by system settings the parameters configuration for operating the network including routing paths between UE and eNB, scheduling, transmission power and MCS assigned to each transmission.

\subsection{Problem Formulation}

The linear program which models the problem, and the column generation algorithm to solve it, are similar to those presented in [8]. The main evolution in the present paper is the Integer Linear Program (ILP) which computes the ISet involved in the optimal solution. In our settings, the ISet generation takes into account the adaptive modulation (multi-rate transmission) and continuous power control functionalities of the nodes in the network.

For sake of space, we present in the following the ISet generation ILP and refer to the aforementioned paper for the technical details. In the column generation algorithm, the following ISet is given as input the graph representing the network together with a weight function on the edges, $\gamma$, and a parameter $\sigma$. Both $\gamma(e), e \in \mathcal{L}$ and $\sigma$ are dual values of the main linear program as explained in [8]. The weight function $\gamma$ gives an incentive to activate the lesser loaded edges while $\sigma$ parametrizes the tradeoff between the throughput provided by the computed ISet and its energy cost. There are only involved in the objective function of the ILP (3) while equations (4) to (7) define the proper structure of an ISet as follows.

$$
\begin{gathered}
\max _{\Psi, P_{t}, J} \sum_{e \in \mathcal{L}}\left(r_{e}^{k} \cdot \gamma(e)\right)-\sigma \sum_{u \in V} J(u) \\
\forall u \in V \quad J(u) \geq \Delta_{p}(u) \cdot \sum_{k \in K_{f}} P_{t}^{k}(u) \\
+\sum_{v \in V} \sum_{\substack{k \in K_{f} \\
i \leq N_{r}}} P_{r}^{k}(u) \cdot \Psi_{(v, u), k}^{i}+P_{0}(u)
\end{gathered}
$$

\footnotetext{
${ }^{2}$ Since the objectives of the minimization of energy consumption and the maximization of network capacity are in conflict with each other, it is not possible to optimize the two objectives in the same time. The tradeoff between them is investigated in Section 4.2
} 


$$
\begin{array}{r}
\forall(u, v) \in \mathcal{L}, i \in\left[1, N_{r}\right], k \in K_{f} \quad P_{t}^{k}(u) \cdot G(u, v) \geq \\
\beta\left(r_{i}\right) \cdot\left(\sum_{u^{\prime} \neq u, v} P_{t}^{k}\left(u^{\prime}\right) \cdot G\left(u^{\prime}, v\right)+\mu\right)-\left(1-\Psi_{(u, v), k}^{i}\right) \cdot n \cdot P_{\max } \\
\forall u \in V, k \in K_{f} \sum_{v \in V} \sum_{1 \leq i \leq N_{r}} \Psi_{(u, v), k}^{i}+\sum_{w \in V} \sum_{1 \leq i \leq N_{r}} \Psi_{(w, u), k}^{i} \leq 1 \\
\forall e=(u, v) \in \mathcal{L}, k \in K_{f} \quad r_{e}^{k}=\sum_{1 \leq i \leq N_{r}} r_{i} \Psi_{(u, v), k}^{i} \\
\forall u \in V \sum_{k \in K_{f}} P_{t}^{k}(u) \leq P_{\max }
\end{array}
$$

The decision variables of this linear program are $P_{t}(u), J(u)$ and $\Psi_{(u, v)}^{i}$ where $(u, v) \in \mathcal{L}$ and $i \in\left[1, N_{r}\right] . J(u)$ is the energy consumption of the node $u$ and $\Psi_{(u, v)}^{i}$ is a binary variable which is equal to 1 if the communication between $u$ and $v$ is active, in the new ISet, with a transmission rate equals at $r_{i}$, and to 0 otherwise. The energy consumption model, detailed in subsection 3.2 , is presented by constraints (4). The constraint (5) ensures that the SINR condition is satisfied for all active links, in the ISet, taking into account the transmission rate used by each one. Note that $\left(1-\Psi_{(u, v)}^{1}\right) \cdot n \cdot P_{\max }$ equals 0 when the link $(u, v)$ is active, hence the constraint (5) reverts back to the classical interferences constraint 11. Otherwise $\left(\Psi_{(u, v)}^{1}=0\right)$, and finally $n \cdot P_{\max }$ ensures that $P_{t}(u)$ can be equal to 0 (constraint (5) is always respected). Finally, constraints (6) and (7) imply that each node is active in at most one link with a single transmission rate in each scheduling block.

\section{Result analysis and discussion}

The simulated network is represented by a hexagonal cellular network composed of three tri-sectored sites. A set of $10 \mathrm{RNs}$ are deployed in each sector in low SNR areas (mainly at the cell border). To study a multi-hop relay network, the RNs are positioned in two circles around the eNB one at $0.4 * I S D$ and other at $0.66 * I S D[6]$. In each macro-cell, 30 users are randomly distributed. A deployment example of a seven macro-cells network is illustrated in Fig 4. In all our scenarios, we assume a quasi-static traffic and quasi time-invariant channel gains and that the system employs an adaptive modulation and coding scheme with 5 discrete rates (and 5 corresponding SNR thresholds) as presented in Table 3. The main parameters are summarized in Table 2 according to $3 \mathrm{GPP}$ LTE-Advanced Case 3 [15.

\subsection{Relay node gain}

In this study, we compute the maximum network capacity and the minimum energy consumption to achieve it as a function of the eNB maximum transmit power per $\mathrm{PRB}\left(P_{\max / P R B}(e N B)\right)$. Fig. 5 depicts the maximum network capacity while Fig. 6 depicts the energy consumption per $\mathrm{Mb} / \mathrm{s}$. In order to 


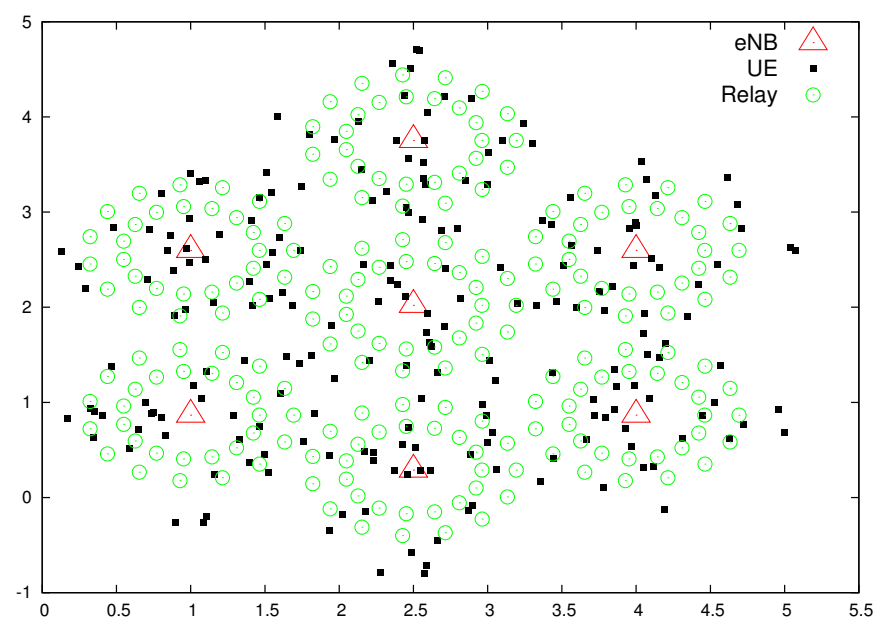

Figure 4: Deployment of eNB and RNs in rural environment: the UEs are randomly distributed in each macro-cell.

Table 2: Simulation parameters assumption

\begin{tabular}{|c|c|}
\hline Parameters & Assumptions \\
\hline Carrier $/$ bandwith & $2 \mathrm{GHz} / 10 \mathrm{MHz}$ \\
\hline PRB bandwith & $180 \mathrm{KHz}$ \\
\hline Noise power density & $9 \mathrm{~dB}(\mathrm{UE}) / 5 \mathrm{~dB}(\mathrm{RN})$ \\
\hline Noise figure & $10(\mathrm{rural})$ \\
\hline Number of RNs & Uniform random distribution \\
\hline User distribution & $1732 \mathrm{~m}(\mathrm{rural})$ \\
\hline ISD (inter-site distance) & quasi-static traffic $(50 \% \mathrm{UL}, 50 \% \mathrm{DL})$ \\
\hline Traffic model & According to $[15$, case $3 \mathrm{rural}$ \\
\hline Pathloss model & $\mathrm{eNB}: A(\theta)=-\min \left[12\left(\frac{\theta}{\theta_{3 d B}}\right)^{2}, A_{m}\right]$ \\
\hline Antenna pattern & $A_{m}=20 d B, \theta_{3 d B}=70^{\circ} ;$ gain $=14 \mathrm{dBi}$ \\
& $\mathrm{RN}:$ Omnidirectional; gain $=5 \mathrm{dBi}$ \\
\hline Maximum transmit power & eNB:46dBm \\
& $\mathrm{RN}: 30 \mathrm{dBm}$ \\
& $\mathrm{UE}: 23 \mathrm{dBm}$ \\
\hline
\end{tabular}

quantify the gain of the deployment of RNs in macro-cell, we study two scenarios; In the first one, we assume a classical cellular network without RNs while, in the second one, $10 \mathrm{RNs}$ are deployed by sector (we refer to $3 \mathrm{GPP}$ scenarios in rural environment). Fig. 5 shows, first, that the network capacity increases with the transmission power allowed at each PRB. Indeed, increasing the transmit power improves the radio link quality and hence allows to transmit with higher transmission rate. Second, it shows an important gain due to the deployment of RNs. In fact, the distance between RN and associated UE is much smaller than the distance between UE and the eNB. This improves the channel gain (the 
Table 3: Modulation and Coding Schemes: MCS 18

\begin{tabular}{|c|c|c|c|c|c|}
\hline MCS & Modulation & CR & $\beta[d B]$ & Throughput & Efficiency \\
\hline \hline MCS1 & QPSK & $1 / 2$ & 1 & $164 \mathrm{~Kb} / \mathrm{s}$ & $0.933 \mathrm{~b} / \mathrm{s} / \mathrm{Hz}$ \\
\hline MCS2 & 16QAM & $1 / 2$ & 10 & $328.12 \mathrm{~Kb} / \mathrm{s}$ & $1.866 \mathrm{~b} / \mathrm{s} / \mathrm{Hz}$ \\
\hline MCS3 & 16QAM & $3 / 5$ & 11.40 & $393.75 \mathrm{~Kb} / \mathrm{s}$ & $2.24 \mathrm{~b} / \mathrm{s} / \mathrm{Hz}$ \\
\hline MCS4 & 64QAM & $1 / 2$ & 11.80 & $492.18 \mathrm{~Kb} / \mathrm{s}$ & $2.8 \mathrm{~b} / \mathrm{s} / \mathrm{Hz}$ \\
\hline MCS5 & 64QAM & $3 / 5$ & 13.80 & $590.625 \mathrm{~Kb} / \mathrm{s}$ & $3.36 \mathrm{~b} / \mathrm{s} / \mathrm{Hz}$ \\
\hline
\end{tabular}

path loss is reduced) and enables a better spatial reuse which allows multiple transmissions to take place simultaneously in the same cell.

For each value of $P_{\max / P R B}$, the energy consumption to provide $1 \mathrm{Mb} / \mathrm{s}$ of the maximum network capacity is reported in Fig. 6. It shows a near-linear growth of the energy consumption with the transmission power. It also shows the energy efficiency of the RNs deployment, particularly, when the transmission power is high (which provide a high network capacity). In fact, a RN consumes much lower than an eNB, in particular when communicating with cell edge users. Hence, because the energy consumption of eNB is near-linear with its transmission power, to activate the RNs and to reduce the transmission power of the eNB provides more energy efficiency. When the transmission power is low the scenario with RNs consumes more because the fixed energy consumption $P_{0}(R N)$ is more expensive than the gain due to the reduction of the transmission power of eNB. In order to assess this conclusion, the energy consumption of the system assuming that there is no fixed energy cost of RNs is reported as "With $R N, P_{0}(R N)=0$ " whose growth is near linear with the transmit power. As a matter of fact, the difference between fixed cost and null fixed cost cases evolves as $\frac{1}{P}$ because the fixed costs are actually "mutualized" among the elements of capacity that are provided. A system-wide challenge is then to reduce the magnitude of the fixed costs, by optimizing the electronic components or by implementing sleep mode of RNs as proposed in [1] 19].

Overall, both the energy consumption and the network capacity increase with the transmission power. This highlights implicitly the existence of a possible energy-capacity tradeoff as detailed in the following.

\subsection{Energy-capacity Tradeoff}

Fig. 7 depicts the energy-capacity Pareto front of a heterogenous cellular network. The energy consumption is presented as $\mathrm{J} / \mathrm{bit}$, obtained by divided the total power consumption (Watts) by the network capacity (bits/s). First, we note the existence of a minimal energy consumption required to meet the capacity constraint: if less energy is available, the traffic demand of UEs can not be served. Second, the capacity tends to a maximum boundary. Between those two points, the capacity increases with the energy consumption. This tradeoff is the result of the use of different MCS and the impact of the spatial reuse. In fact, optimizing the capacity needs to increase the number of simultaneous communications (spatial reuse) and to use high transmission rate, but this increases the transmission power cost in order to mitigate the resulting interferences and to ensure that the SINR threshold is satisfied for all active links. The minimum 


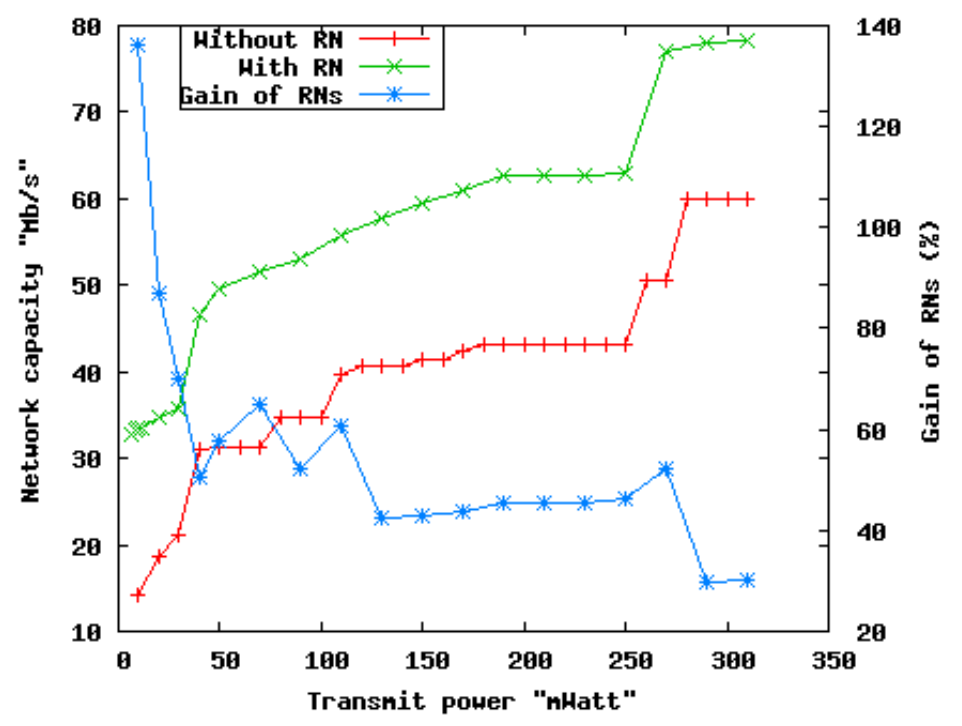

Figure 5: Gain of network capacity due to relay distribution.

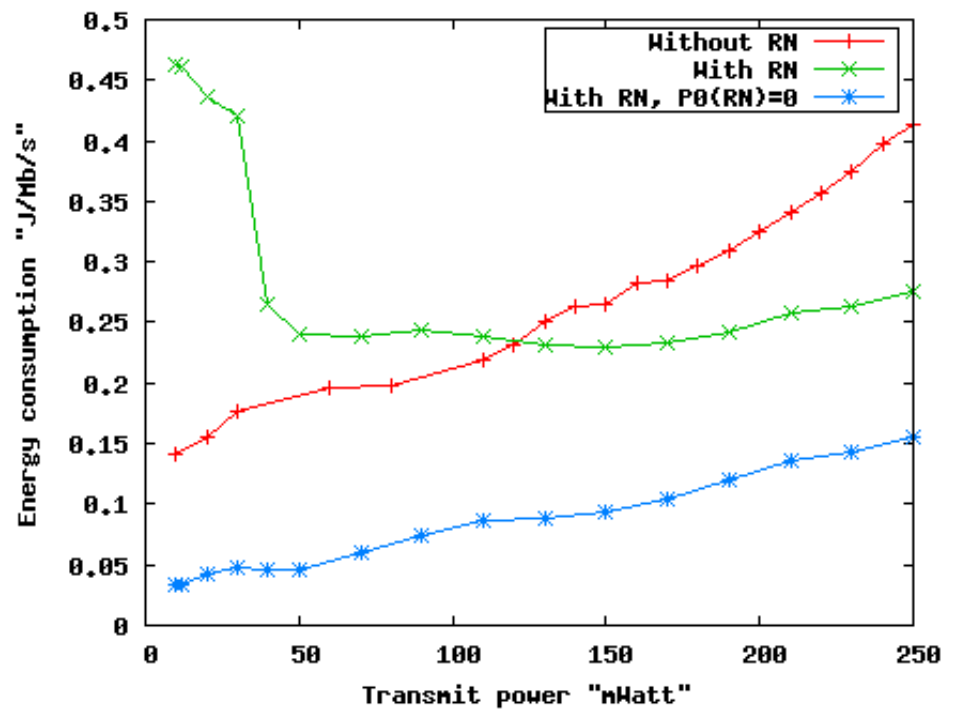

Figure 6: Gain of network capacity due to relay distribution.

energy consumption is obtained when only one communication is active in a scheduling block (SB) using the lowest transmission rate.

\subsection{Multi-hop mesh backhauling network}

Based on the optimal network configuration (in particular the paths between UE and eNB), we studied the opportunity of a multi-hop mesh backhauling network among the RNs. We remember that an UE can communicate directly with the eNB or through a multi-hop relay, see Fig. 1. Ours results show that 
the maximum network capacity with efficient energy consumption is obtained with no more than two hops between UE and eNB. This can be explained by the huge interferences generated by the eNB-RN communications which prohibit the other communications between RNs to be activated in the same SB. Therefore, there is no spatial reuse in the backhauling if the eNB is activated. This makes multi-hop communications, in the backhauling, waste the gain in capacity obtained by the relaying.

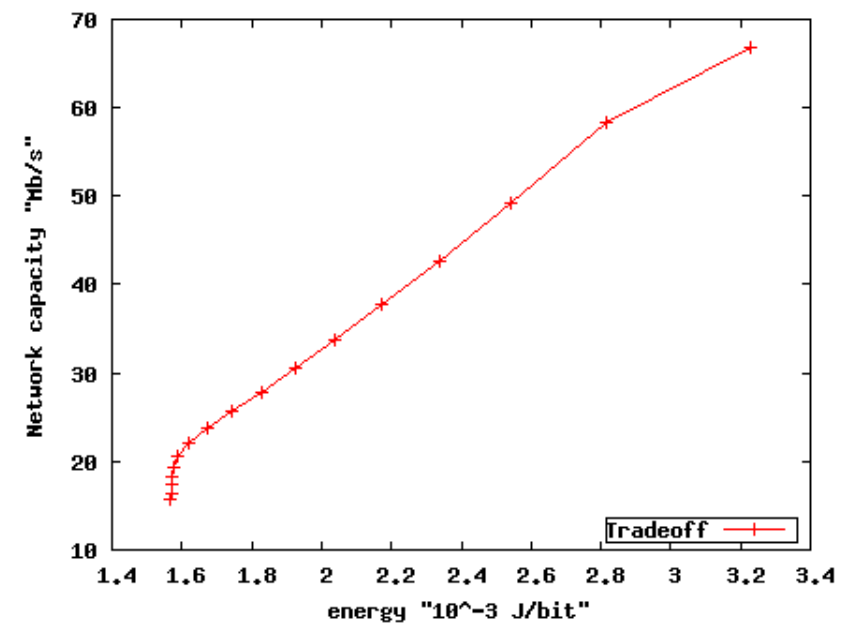

Figure 7: Energy-capacity tradeoff.

\section{Conclusion}

In this paper, we presented an optimization framework used to calculate an optimal offline configuration of heterogeneous cellular network like LTE-Advanced relay that consists of a macro-cell and a certain number of relay nodes. This framework allows us to calculate a joint optimization of resource allocation and users attachment where the goal is to maximize the network capacity with low energy consumption. Our computational results show a significant gain in throughput and energy consumption due to the deployment of the RNs. They also show an important tradeoff between maximizing the network capacity and minimizing the energy consumption. A deeper challenge is to develop a protocol or distributed algorithm based on the results of our study which provides high network capacity with low energy consumption.

\section{Acknowledgment}

This work has been partially funded by the ANR VERSO project ECOSCells. 


\section{References}

[1] Pål K. Frenger, Peter Moberg, Jens Malmodin, Ylva Jading, and István Gódor. Reducing energy consumption in lte with cell dtx. In VTC Spring'11, pages 1-5, 2011.

[2] ECOSCells. Efficient COoperating Small Cells. November 2009.

[3] Anton Ambrosy and Project Partners. Definition and parameterization of reference system and scenarios. INFSO-ICT-247733 EARTH, Deliverable D2.2, June 2010 .

[4] 3GPP R1-104460. Type-1 relay performance for uplink.

[5] 3GPP TR 36.921. Evolved universal terrestrial radio access (e-utra); fdd home enode b (henb) radio frequency (rf) requirements analysis. Technical report, Avril 2010 .

[6] Bin Fan and Saadani Ahmed. Full reuse resource partition for multihop relay networks with tree and mesh structures. In IEEE 22nd International Symposium on Personal Indoor and Mobile Radio Communications (PIMRC), 2011, Toronto, Canada, September 2011.

[7] J. Ghimire and C. Rosenberg. On the need for coordination among base stations in a heterogeneous network. In IEEE 2012 International Symposium of Modeling and Optimization in Mobile, Ad Hoc, and Wireless Networks (WiOpt 2012), Paderborn, Germany, May 2012.

[8] Anis Ouni, Hervé Rivano, and Fabrice Valois. Wireless Mesh Networks: Energy - Capacity Tradeoff and Physical Layer Parameters. In 22nd IEEE International Symposium on Personal, Indoor and Mobile Radio Communications (PIMRC), Toronto, Canada, September 2011. IEEE.

[9] Kevin R. Jacobson and Witold A. Krzymien. Realistic throughput of cellular multi-hop relay networks with spatial reuse. WPMC, 2006.

[10] Roberto Fantini, Dario Sabella, and Marco Caretti. Energy efficiency in lteadvanced networks with relay nodes. In IEEE 73rd Vehicular Technology Conference (VTC Spring), Budapest, Hungary, 15-18 May 2011.

[11] Abdallah Bou Saleh, Ömer Bulakci, Simone Redana, Bernhard Raaf, and Jyri Hämäläinen. Evaluating the energy efficiency of lte-advanced relay and picocell deployments. In IEEEWireless Communications and Networking Conference: Mobile and Wireless Networks, pages 2335-2340, Shanghai, 1-4 April 2012.

[12] J. Ghimire and C. Rosenberg. Resource allocation, transmission coordination and user association in heterogeneous networks: a flow-based unified approach. IEEE TWC, December 2012.

[13] EARTH Project. Energy efficiency analysis of the reference systems, areas of improvements and target breakdown. INFSO-ICT-247733 EARTH, Deliverable D2.3, December 2010.

[14] Donghan Chee, Kang Min, Lee Howon, and Jung Bang. A study on the green cellular network with femtocells. In Third International Conference on Ubiquitous and Future Networks (ICUFN), Dalian, China, June 2011. IEEE.

[15] 3GPP TR 36.814 V9.0.0. Technicalspecificationgroupradioaccessnetwork; evolved universal terrestrial radio access (e-utra); further advancements for e-utra physical layer aspects (release 9). Technical report, Technical Report 3GPP, 2010.

[16] R. Fourer, D. M. Gay, and B. W. Kernighan. A modeling language for mathematical programming. Management Science, 36(5):519-554, 1990.

[17] Ilog, Inc. Solver cplex, 2003. 
[18] D. Lopez-Peres, A. Ladanyi, A. Jüttner, H. Rivano, and J. Zhang. Optimization Method for the Joint Allocation of Modulation Schemes, Coding Rates, Resource Blocks and Power in Self-Organizing LTE Networks. In 30th IEEE International Conference on Computer Communications mini-conf, Shanghai, China, April 2011.

[19] Alexandra Bousia, Angelos Antonopoulos, Luis Alonso, and Christos V. Verikoukis. "green" distance-aware base station sleeping algorithm in lte-advanced. In ICC, pages 1347-1351. IEEE, 2012. 


\section{Contents}

$\begin{array}{lll}1 & \text { Introduction } & 3\end{array}$

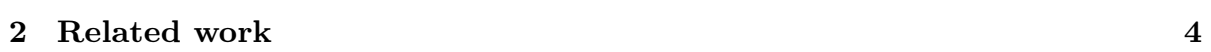

3 System model and Optimization framework 5

3.1 Problem definition and assumptions . . . . . . . . . . . . . 5

3.2 Energy consumption model . . . . . . . . . . . . . . . . . . . 6

3.3 Network model and notations . . . . . . . . . . . . . . . . . . 6

3.4 Optimization tools . . . . . . . . . . . . . . . . . . . 7

3.5 Problem Formulation . . . . . . . . . . . . . . . . . . . . . . . . . . 8

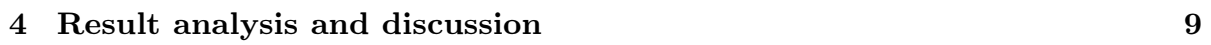

4.1 Relay node gain . . . . . . . . . . . . . . . . . . . . . . . . . 9

4.2 Energy-capacity Tradeoff . . . . . . . . . . . . . . . . . . . . . . . . . 11

4.3 Multi-hop mesh backhauling network . . . . . . . . . . . . . . . . . . 12

$\begin{array}{lll}5 & \text { Conclusion } & 13\end{array}$

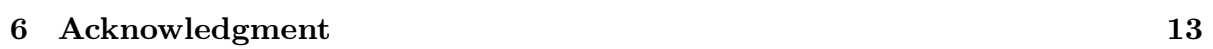


Centre de recherche INRIA Grenoble - Rhône-Alpes 655, avenue de l'Europe - 38334 Montbonnot Saint-Ismier (France)

Centre de recherche INRIA Bordeaux - Sud Ouest : Domaine Universitaire - 351, cours de la Libération - 33405 Talence Cedex Centre de recherche INRIA Lille - Nord Europe : Parc Scientifique de la Haute Borne - 40, avenue Halley - 59650 Villeneuve d'Ascq Centre de recherche INRIA Nancy - Grand Est : LORIA, Technopôle de Nancy-Brabois - Campus scientifique 615, rue du Jardin Botanique - BP 101 - 54602 Villers-lès-Nancy Cedex

Centre de recherche INRIA Paris - Rocquencourt : Domaine de Voluceau - Rocquencourt - BP 105 - 78153 Le Chesnay Cedex

Centre de recherche INRIA Rennes - Bretagne Atlantique : IRISA, Campus universitaire de Beaulieu - 35042 Rennes Cedex Centre de recherche INRIA Saclay - Île-de-France : Parc Orsay Université - ZAC des Vignes : 4, rue Jacques Monod - 91893 Orsay Cedex

Centre de recherche INRIA Sophia Antipolis - Méditerranée : 2004, route des Lucioles - BP 93 - 06902 Sophia Antipolis Cedex 